\title{
LOCAL MONOTONICITY OF RIEMANNIAN AND FINSLER VOLUME WITH RESPECT TO BOUNDARY DISTANCES
}

\author{
SERGEI IVANOV
}

\begin{abstract}
We show that the volume of a simple Riemannian metric on $D^{n}$ is locally monotone with respect to its boundary distance function. Namely if $g$ is a simple metric on $D^{n}$ and $g^{\prime}$ is sufficiently close to $g$ and induces boundary distances greater or equal to those of $g$, then $\operatorname{vol}\left(D^{n}, g^{\prime}\right) \geq \operatorname{vol}\left(D^{n}, g\right)$. Furthermore, the same holds for Finsler metrics and the Holmes-Thompson definition of volume. As an application, we give a new proof of injectivity of the geodesic ray transform for a simple Finsler metric.
\end{abstract}

\section{INTRODUCTION}

A Riemannian metric $g$ on the $n$-dimensional disc $D=D^{n}$ is called simple if the boundary $\partial D$ is strictly convex with respect to $g$ (that is, its second fundamental form is positive definite) and all geodesics in $(D, g)$ are minimizing and have no conjugate points (or, equivalently, every pair of points in $D$ is connected by a unique geodesic which varies smoothly with the endpoints). Note that this property persists under $C^{\infty}$-small perturbations of the metric.

For a Riemannian metric $g$ on $D$, we denote by $d_{g}$ the distance function on $D \times D$ induced by $g$. The boundary distance function of $g$, denoted by $b d_{g}$, is the restriction of $d_{g}$ to $\partial D \times \partial D$. That is, $b d_{g}(x, y)$ is the length of a $g$-shortest path in $D$ between boundary points $x$ and $y$. If $g$ is simple, this shortest path is the (unique) $g$-geodesic connecting $x$ and $y$.

It is well-known that the volume of a simple metric $g$ is determined by the function $b d_{g}$ via an explicit formula involving boundary distances and their derivatives (cf. [21, [11, [8]). It is natural to expect that this formula is monotone with respect to $b d_{g}$, that is, if another metric $g^{\prime}$ satisfies $b d_{g^{\prime}} \geq b d_{g}$ pointwise, then $\operatorname{vol}\left(D, g^{\prime}\right) \geq \operatorname{vol}(D, g)$. However the formula itself is not monotone if arbitrary functions are allowed in place of $b d_{g}$ (see Example 2.3). On the other hand, functions that can be realized by boundary distances of simple metrics are rather special, and it might be the case that the volume formula is monotone within this class of functions.

This question is a variant of the minimal filling conjecture (see [5, 15]) which asserts that a simple Riemannian metric $g$ has the least volume among all metrics whose boundary distance functions majorize that of $g$. This conjecture is related to Michel's boundary rigidity conjecture [17] about unique determination of a simple metric by its boundary distance function.

2010 Mathematics Subject Classification. 53C60, 53C20, 44A12.

Key words and phrases. Boundary distance function, minimal filling, Finsler metric, HolmesThompson volume, geodesic ray transform.

Supported by RFBR grants 09-01-12130-ofi-m and 11-01-00302-a. 
The minimal filling conjecture is essentially about finding metrics realizing filling volumes, see [11. It has been confirmed in a number of special cases. In dimension 2 the conjecture is proved for any simple metric $g$ and any competing metric $g^{\prime}$ on $D^{2}$ (13], see also [14] for the Finslerian case and [20] for boundary rigidity). However the general case where the competing metric $g^{\prime}$ can be on a surface of arbitrary genus remains open, even for $\left(D^{2}, g\right)$ isometric to a subset of the standard hemisphere. (The latter special case is equivalent to Gromov's Filling Area Conjecture.) In higher dimensions, filling minimality is established for flat metrics by Gromov [11, for regions in negatively curved symmetric spaces by Besson, Courtois and Gallot [4, and for metrics $g$ sufficiently close to a flat or hyperbolic metric by Burago and Ivanov [5, 6]. These results come with equality case analysis that yields boundary rigidity of the respective metrics. Croke and Kleiner 10 . proved boundary rigidity of some product metrics using a weaker form of volume minimality. Croke, Dairbekov and Sharafutdinov [9] proved local filling minimality and boundary rigidity for metrics with certain upper curvature bounds.

In this paper we settle the local version of the minimal filling conjecture, namely we prove the following theorem.

Theorem 1. For every simple Riemannian metric $g_{0}$ on $D^{n}$ there is a neighborhood $\mathcal{U}$ of $g_{0}$ in the space of all Riemannian metrics on $D^{n}$ (with the $C^{\infty}$ topology) such that the following holds. For all metrics $g, g^{\prime} \in \mathcal{U}$ such that

$$
d_{g^{\prime}}(x, y) \geq d_{g}(x, y) \quad \text { for all } x, y \in \partial D,
$$

one has

$$
\operatorname{vol}\left(D^{n}, g^{\prime}\right) \geq \operatorname{vol}\left(D^{n}, g\right) .
$$

The same result holds for Finsler metrics (see Theorem 2 below), and even in the Riemannian case the proof relies on Finsler geometry. The plan of the proof is the following. First we show that the fact that boundary distances determine the volume extends to Finsler metrics. Then, for metrics $g$ and $g^{\prime}$ as in Theorem 1 we construct a (non-reversible) Finsler metric $\varphi$ on $D$ which induces the same boundary distances as $g^{\prime}$ and majorizes $g$ pointwise in $D$. It then follows that $\operatorname{vol}\left(D, g^{\prime}\right)=\operatorname{vol}(D, \varphi) \geq \operatorname{vol}(D, g)$. Finsler metrics help here because they are flexible: unlike in the Riemannian case, it is easy to construct perturbations of a Finsler metric that induce a given perturbation of the boundary distance function. The actual details of the proof are more complicated than the above plan: to work around non-smoothness of the boundary distance function at the diagonal, we extend the metric to a slightly larger disc and use distances in that larger disc rather than the original one, see section 2 ,

Let us proceed with definitions and formulations for the Finslerian case. A Finsler manifold is a smooth manifold equipped with a Finsler metric. A Finsler metric on a smooth manifold $M$ is a continuous function $\varphi: T M \rightarrow \mathbb{R}$ satisfying the following conditions:

(1) $\varphi(t v)=t \varphi(v)$ for all $v \in T M$ and $t \geq 0$;

(2) $\varphi$ is positive on $T M \backslash 0$;

(3) $\varphi$ is smooth on $T M \backslash 0$;

(4) $\varphi$ is strictly convex in the following sense: for every $x \in M$, the function $\varphi_{x}:=\left.\varphi^{2}\right|_{T_{x} M}$ has positive definite second derivatives on $T_{x} M \backslash\{0\}$.

Note that we do not require that $\varphi(-v)=\varphi(v)$, that is, non-reversible Finsler metrics are allowed. Nevertheless we still referq to functions $\varphi_{x}$ as norms on the 
fibers $T_{x} M$. For a Finsler metric $\varphi$, one naturally defines geodesics, lengths, and a (non-symmetric) distance function $d_{\varphi}: M \times M \rightarrow \mathbb{R}_{+}$, see e.g. [1] for details. We define the notion of a simple Finsler metric and the boundary distance function $b d_{\varphi}$ in the same way as in the Riemannian case.

For the notion of volume of a Finsler metric we use the Holmes-Thompson definition [12, reproduced here for the reader's convenience. Let $M=(M, \varphi)$ be a Finsler manifold. Consider the co-tangent bundle $T^{*} M$ and let $\varphi^{*}: T^{*} M \rightarrow \mathbb{R}$ be the fiber-wise dual norm to $\varphi$. That is, for $x \in M$ and $\alpha \in T_{x}^{*} M$, one defines

$$
\varphi^{*}(\alpha)=\sup \left\{\alpha(v) \mid v \in T_{x} M, \varphi(v)=1\right\} .
$$

Let $B^{*} M=B^{*}(M, \varphi)$ the bundle of unit balls of $\varphi^{*}$ :

$$
B^{*} M=\left\{\alpha \in T^{*} M \mid \varphi^{*}(\alpha) \leq 1\right\} .
$$

The Holmes-Thompson volume of $M$, that we denote by $\operatorname{vol}(M) \operatorname{or} \operatorname{vol}(M, \varphi)$, is defined by

$$
\operatorname{vol}(M)=\frac{1}{\omega_{n}} \operatorname{Vol}_{c a n}\left(B^{*} M\right)
$$

where $n=\operatorname{dim} M, \omega_{n}$ is the Euclidean volume of the unit ball in $\mathbb{R}^{n}$, and $\operatorname{Vol}_{\text {can }}$ is the canonical (symplectic) $2 n$-dimensional volume on $T^{*} M$. Clearly this definition yields the Riemannian volume in the case when the Finsler metric is Riemannian. Also notice that the volume is monotonous with respect to the metric: if $\varphi^{\prime} \geq \varphi$ pointwise, then $B^{*}\left(M, \varphi^{\prime}\right) \supset B^{*}(M, \varphi)$ and therefore $\operatorname{vol}\left(M, \varphi^{\prime}\right) \geq \operatorname{vol}(M, \varphi)$.

Theorem 2. For every simple Finsler metric $\varphi_{0}$ on $D^{n}$ there is a $C^{\infty}$ neighborhood $\mathcal{U}$ of $\varphi_{0}$ such that the following holds. For all Finsler metrics $\varphi, \varphi^{\prime} \in \mathcal{U}$ such that $b d_{\varphi^{\prime}} \geq b d_{\varphi}$ pointwise, one has $\operatorname{vol}\left(D^{n}, \varphi^{\prime}\right) \geq \operatorname{vol}\left(D^{n}, \varphi\right)$.

Remark 1.1. Similar Finsler volume comparison results (in the reversible case) were obtained recently by H. Koehler [16. In particular, Corollary 3.2(2) in 16 asserts filling minimality of $\varphi$ among all simple metrics $\varphi^{\prime}$ such that $b d_{\varphi^{\prime}}$ is sufficiently close to $b d_{\varphi}$ in the strong $C^{2}$ topology (in the complement of the diagonal in $\partial D \times \partial D$ ). This result implies the assertion of Theorem 2 under an additional assumption that $\varphi$ and $\varphi^{\prime}$ (along with their derivatives up to a certain order) agree on $\partial D$.

Remark 1.2. Finsler metrics are never boundary rigid as they admit non-isometric perturbations preserving boundary distances. (One possible construction is described in section 2, see the paragraph preceding Lemma 2.2.) Because of this, our Finslerian proof of Theorem 1 does not have immediate rigidity implications.

Theorem 1 is a special case of Theorem 2 The proof of Theorem 2 occupies sections 2 and 3 with Appendix $\mathrm{A}$ containing the proof of a technical lemma.

In section 4 we show how Theorem 2 implies the (well-known) injectivity of the geodesic ray transform for a simple Finsler metric, see Corollary 1, Loosely speaking, this injectivity means that a smooth function on $D$ is uniquely determined by its integrals over geodesics. More precisely, consider a simple Finsler metric $\varphi$ on $D=D^{n}$ and denote by $\Gamma_{\varphi}$ the space of all maximal geodesics of this metric (this space is a $(2 n-2)$-dimensional smooth manifold diffeomorphic to the complement of the diagonal in $\partial D \times \partial D)$. The geodesic ray transform of $\varphi$ is a map

$$
I_{\varphi}: C^{\infty}(D) \rightarrow C^{\infty}\left(\Gamma_{\varphi}\right)
$$


defined by

$$
I_{\varphi} f(\gamma)=\int_{\operatorname{dom}(\gamma)} f(\gamma(t)) d t \quad f \in C^{\infty}(D), \gamma \in \Gamma_{\varphi}
$$

(Here and everywhere in the paper the geodesics are parametrized by arc length, that is, $\varphi(\dot{\gamma}(t)) \equiv 1$ for all $\gamma \in \Gamma_{\varphi}$ and $t \in \operatorname{dom}(\gamma)$.)

Corollary 1. If $n \geq 2$ and $\varphi$ is a simple Finsler metric on $D^{n}$, then $I_{\varphi}$ is injective.

This result is not new; a more general theorem is proved by Sharafutdinov [22] by analytic methods. For Riemannian metrics, the injectivity of the geodesic ray transform for a simple metric is proved by Mukhometov [18, 19] and independently by Bernstein and Gerver [2, 3].

Corollary 1 easily follows from Theorem 2 applied to metrics conformal to $\varphi$, see section 4 for details. The author believes that this new proof is more geometric and transparent than the one in 22 .

Acknowledgement. The author is grateful to V. A. Sharafutdinov and G. Uhlmann for useful discussion of the history of the geodesic ray transform problem.

\section{ENVELOPING FUNCTIONS}

Let $\varphi$ be a Finsler metric on $D=D^{n}$. We denote by $U D$ and $U^{*} D$ the bundles of unit spheres of $\varphi$ and $\varphi^{*}$, respectively. To emphasize the dependence on $\varphi$ where needed, we write $U(D, \varphi)$ and $U^{*}(D, \varphi)$. We say that a smooth function $f: D \rightarrow \mathbb{R}$ is distance-like (with respect to $\varphi$ ) if $\varphi^{*}\left(d_{x} f\right)=1$ for all $x \in D$.

If $\varphi$ is simple, then the distance function $d_{\varphi}(p, \cdot)$ of a point $p \in D$ satisfies this requirement everywhere except at $p$. To construct a smooth distance-like function, consider a larger disc $D^{+} \supset D$ and smoothly extend the metric $\varphi$ to it. For a fixed extension, choosing $D^{+}$sufficiently close to $D$ guarantees that $\varphi$ is simple on $D^{+}$. Then for every $p \in \partial D^{+}$the function $\left.d_{\varphi}(p, \cdot)\right|_{D}$ is smooth and distance-like.

The (Finslerian) gradient of a distance-like function $f: D \rightarrow \mathbb{R}$ at $x \in D$, denoted by $\operatorname{grad}_{\varphi} f(x)$, is the unique tangent vector $v \in U_{x} D$ such that $d_{x} f(v)=1$. In other words, $\operatorname{grad}_{\varphi} f(x)$ is the Legendre transform of the co-vector $d_{x} f$ with respect to the Lagrangian $\frac{1}{2} \varphi^{2}$.

For example, the gradient at $x$ of the distance function $d_{\varphi}(p, \cdot)$ of a simple Finsler metric $\varphi$ is the velocity at the endpoint of the unique minimizing geodesic from $p$ to $x$. We denote this velocity vector by $\overleftarrow{x p}$.

A gradient curve of $f$ is a curve $\gamma:[a, b] \rightarrow D$ such that $\dot{\gamma}(t)=\operatorname{grad}_{\varphi}(\gamma(t))$ for all $t \in[a, b]$. Clearly every gradient curve of a distance-like function is a minimizing geodesic.

Definition 2.1. Fix a manifold $S$ diffeomorphic to $S^{n-1}$. We say that a smooth function $F: S \times D \rightarrow \mathbb{R}$ is an enveloping function for $\varphi$ if the following two conditions are satisfied:

(i) for every $p \in S$, the function $F_{p}:=F(p, \cdot)$ is distance-like;

(ii) for every $x \in D$, the map $p \mapsto d_{x} F_{p}$ is a diffeomorphism from $S$ to $U_{x}^{*} D$.

We construct an enveloping function for a simple metric $\varphi$ as follows. Consider a disc $D^{+} \supset D$ with metric $\varphi$ extended as above. Identify $S$ with $\partial D^{+}$and define

$$
F(p, x)=F_{p}(x)=d_{\varphi}(p, x)
$$


for all $p \in S=\partial D^{+}$and $x \in D$. Then $F$ is an enveloping function. Indeed, for a fixed $x \in D$, the map $p \mapsto \operatorname{grad}_{\varphi} F_{p}(x)=\overleftarrow{x p}$ is a diffeomorphism between $S$ and $U_{x} D$ because the metric is simple. Since the Legendre transform (from $U_{x} D$ to $\left.U_{x}^{*} D\right)$ is also a diffeomorphism, so is the map $p \mapsto d_{x} F_{p}$ from $S$ to $U_{x}^{*} D$.

Some non-simple metrics admit enveloping functions as well. For example, if $F: S \times D \rightarrow \mathbb{R}$ is an enveloping function for a metric $\varphi$ on $D$, and $D^{\prime} \subset D$ is a sub-domain with smooth (but not necessarily convex) boundary, then $\left.F\right|_{S \times D^{\prime}}$ is an enveloping function for $\left.\varphi\right|_{D^{\prime}}$. However the existence of an enveloping function implies that all geodesics are minimizing (because they are gradient curves of distance-like functions) and therefore have no conjugate points.

An enveloping function $F$ uniquely determines the metric. Indeed, the unit sphere of the dual norm $\varphi^{*}$ at every point $x \in D$ is the image of the map $p \mapsto d_{x} F_{p}$ from $S$ to $T_{x}^{*} D$ and thus is determined by $F$. This unit sphere determines the dual norm $\varphi_{x}^{*}$ and the latter determines the Finsler norm $\varphi_{x}$.

Furthermore every $C^{3}$-small perturbation of $F$ yields an enveloping function of a Finsler metric. Indeed, let $F$ be an enveloping function for $\varphi$ and $F^{\prime}: S \times D \rightarrow \mathbb{R}$ be $C^{3}$-close to $F$. Define $F_{p}^{\prime}=F^{\prime}(p, \cdot)$ for every $p \in S$. Then for every $x \in D$, the map $p \mapsto d_{x} F_{p}^{\prime}$ is $C^{2}$-close to the similar map for $F$. Therefore the image of this map is a convex surface in $T^{*} M$, and this surface is the unit sphere of some norm $\varphi_{x}^{\prime *}$. The dual norm to $\varphi_{x}^{\prime *}$ is a norm $\varphi_{x}^{\prime}$ on $T_{x} M$, and the union of these norms over all $x \in D$ is a Finsler metric $\varphi^{\prime}$ for which $F^{\prime}$ is an enveloping function.

The boundary distance function of a simple metric $\varphi$ is uniquely determined by the restriction $\left.F\right|_{S \times \partial D}$ of an enveloping function $F$ to the boundary. Namely,

$$
d_{\varphi}(x, y)=\max _{p \in S}(F(p, y)-F(p, x))
$$

for $x, y \in \partial D$. (The maximum is attained at a point $p \in \partial D$ such that the minimizing geodesic from $x$ to $y$ is a gradient curve of $F_{p}$.) Therefore every $C^{3}$-small perturbation of $F$ in the interior of its domain produces a perturbation of the metric preserving the boundary distance function.

Lemma 2.2. Let $F$ be an enveloping function for $\varphi$. Then $\operatorname{vol}(D, \varphi)$ is uniquely determined by the restriction $\left.F\right|_{S \times \partial D}$.

Note that in this lemma we do not assume that $\varphi$ is simple. However, as explained above, existence of an enveloping function implies simplicity of the metric provided that the boundary is strictly convex. One could deduce the lemma from the fact that the boundary distance function of a simple Finsler metric uniquely determines the volume, but the author is not aware of a published proof of this fact.

Proof of Lemma 2.2. For every $x \in D$, define a differential $(n-1)$-form $V_{x}$ on $U_{x}^{*} D$ with values in $\Lambda^{n} T_{x}^{*} D$ as follows: for $\alpha \in U_{x}^{*} D$ and $\eta \in \Lambda^{n-1} T_{v}\left(U_{x}^{*} D\right)$, let

$$
V_{x}(\alpha)(\eta)=\frac{1}{n} \cdot \alpha \wedge i_{*}(\eta)
$$

where $i: U_{x}^{*} D \rightarrow T_{x}^{*} D$ is the inclusion map and $i_{*}: \Lambda^{n-1} T_{v}\left(U_{x}^{*} D\right) \rightarrow \Lambda^{n-1} T_{x}^{*} D$ the induced mapping of $(n-1)$-vectors. The integral of $V_{x}$ over $U_{x}^{*} D$ equals the volume of the unit co-tangent ball $B_{x}^{*}$ (note that the volume of a subset of $T_{x}^{*} D$ has an invariant meaning as an element of $\left.\Lambda^{n} T_{x}^{*} D\right)$. Thus the definition of the 
Holmes-Thompson volume can be written in the form

$$
\operatorname{vol}(D, \varphi)=\frac{1}{\omega_{n}} \int_{D}\left(x \mapsto \int_{U_{x}^{*} D} V_{x}\right)
$$

Let $F: S \times D \rightarrow \mathbb{R}$ be an enveloping function for $\varphi$. For $x \in D$, define a map $G_{x}: S \rightarrow U_{x}^{*} D$ by

$$
G_{x}(p)=d_{x} F_{p}
$$

where $F_{p}=F(p, \cdot)$. By the second requirement of Definition 2.1. $G_{x}$ is a diffeomorphism. It induces a map $G_{x}^{*}: \Lambda^{n-1} T^{*} U_{x}^{*} \rightarrow \Lambda^{n-1} T^{*} S$ which carries differential $(n-1)$-forms from $U_{x}^{*}$ to $S$. From (2.1) we have

$$
\operatorname{vol}(D, \varphi)=\frac{1}{\omega_{n}} \int_{D}\left(x \mapsto \int_{S} G_{x}^{*} V_{x}\right)=\frac{1}{\omega_{n}} \int_{S} \lambda
$$

where $\lambda$ is a differential $(n-1)$-form on $S$ given by

$$
\lambda=\int_{D}\left(x \mapsto G_{x}^{*} V_{x}\right) .
$$

We are going to show that the value $\lambda(p)$ of $\lambda$ at every point $p \in S$ is determined by the restriction $\left.F\right|_{S \times \partial D}$. We have $\lambda(p)=\int_{D} \nu_{p}$ where $\nu_{p}$ is a differential $(n-1)$-form on $M$ with values in $\Lambda^{n-1} T_{p}^{*} S$ defined by

$$
\nu_{p}(x)=\left(G_{x}^{*} V_{x}\right)(p) .
$$

Then for tangent vectors $\xi_{1}, \ldots, \xi_{n-1} \in T_{p} S$ we have

$$
\begin{aligned}
\nu_{p}(x)\left(\xi_{1} \wedge \cdots \wedge \xi_{n-1}\right) & =V_{x}\left(d G_{x}\left(\xi_{1}\right) \wedge \cdots \wedge d G_{x}\left(\xi_{n-1}\right)\right) \\
& =\frac{1}{n} \cdot G_{x}(p) \wedge d G_{x}\left(\xi_{1}\right) \wedge \cdots \wedge d G_{x}\left(\xi_{n-1}\right)
\end{aligned}
$$

where the vectors $d G_{x}\left(\xi_{i}\right)$ in the right-hand side are regarded as elements of $T_{x}^{*} D$ (we implicitly use the inclusion of $T_{v} U^{*} D$ into $T_{x}^{*} D$ for $v=G_{x}(p)$ here). Substituting the definition of $G_{x}$ yields

$$
\nu_{p}(x)\left(\xi_{1} \wedge \cdots \wedge \xi_{n-1}\right)=\frac{1}{n} \cdot d_{x} F_{p} \wedge d_{x} F_{p, \xi_{1}} \wedge \cdots \wedge d_{x} F_{p, \xi_{n-1}}
$$

where $F_{p, \xi_{i}}$ denotes the derivative of $F$ with respect to the first argument along the tangent vector $\xi_{i}$ at $p$. Thus

$$
\lambda(p)\left(\xi_{1} \wedge \cdots \wedge \xi_{n-1}\right)=\frac{1}{n} \int_{D} d F_{p} \wedge d F_{p, \xi_{1}} \wedge \cdots \wedge d F_{p, \xi_{n-1}} .
$$

The $n$-form under the integral in the right-hand side equals the exterior derivative of the $(n-1)$-form $F_{p} \cdot d F_{p, \xi_{1}} \wedge \cdots \wedge d F_{p, \xi_{n-1}}$. Therefore by Stokes' theorem

$$
\lambda(p)\left(\xi_{1} \wedge \cdots \wedge \xi_{n-1}\right)=\frac{1}{n} \int_{\partial D} F_{p} \cdot d F_{p, \xi_{1}} \wedge \cdots \wedge d F_{p, \xi_{n-1}} .
$$

The right-hand side is determined by the restriction $\left.F\right|_{S \times \partial D}$, hence so are $\lambda(p)$ and the volume $\operatorname{vol}(D, \varphi)$ which is written in terms of $\lambda$ in (2.2).

Example 2.3. Consider the case of dimension $n=2$. As the above proof shows, the volume can be expressed in terms of an enveloping function $F=F(p, x)$ restricted on $S \times \partial D$ as follows:

$$
\operatorname{vol}(D, \varphi)=\frac{1}{2 \pi} \int_{S} \int_{\partial D} F(p, x) \cdot \frac{\partial^{2} F}{\partial p \partial x}(p, x) d x d p=-\frac{1}{2 \pi} \int_{S \times \partial D} \frac{\partial F}{\partial x} \cdot \frac{\partial F}{\partial p} d x d p .
$$


Let $f=b d_{\varphi}$. We use the notation $x$ and $y$ for the arguments of $f$. Since $f$ is a limit of enveloping functions (whose first derivatives converge a.e.), we can use the same formula with $f$ in place of $F$ (and $\partial D$ in place of $S$ ):

$$
\operatorname{vol}(D, \varphi)=-\frac{1}{2 \pi} \int_{\partial D \times \partial D} \frac{\partial f}{\partial x} \cdot \frac{\partial f}{\partial y} d x d y .
$$

This is an explicit formula for the volume in terms of the boundary distance function in dimension 2.

Identify $\partial D$ with the standard circle (of length $2 \pi$ ). Let us further restrict ourselves to the case when $f$ is symmetric and invariant under rotations of the circle. Then $\rho$ is determined by a function $f_{0}=f\left(x_{0}, \cdot\right)$ in one variable which ranges over a half-circle. Identifying the half-circle with the segment $[0, \pi]$, we can rewrite (2.3) as follows:

$$
\operatorname{vol}(D, \varphi)=2 \int_{0}^{\pi}\left(\dot{f}_{0}\right)^{2}
$$

It is easy to see that this formula is not monotone with respect to $f_{0}$, even within the class of increasing functions satisfying the triangle inequality. However it is monotone within the class of concave functions, and any rotation-invariant boundary distance function is concave.

\section{Proof of Theorem 2}

Fix a simple Finsler metric $\varphi_{0}$ on $D$ and let $\varphi, \varphi^{\prime}$ be Finsler metrics $C^{\infty}$-close to $\varphi_{0}$ and such that $b d_{\varphi^{\prime}} \geq b d_{\varphi}$. Since simplicity of a metric is an open condition, we may assume that $\varphi$ and $\varphi^{\prime}$ are simple. As explained in the previous section, extend $\varphi$ and $\varphi^{\prime}$ to a larger disc $D^{+} \supset D$ and define enveloping functions

$$
F_{0}, F, F^{\prime}: S \times D \rightarrow \mathbb{R}
$$

for $\varphi_{0}, \varphi$ and $\varphi^{\prime}$ respectively, by

$$
F_{0}(p, x)=d_{\varphi_{0}}(p, x), \quad F(p, x)=d_{\varphi}(p, x), \quad F^{\prime}(p, x)=d_{\varphi^{\prime}}(p, x)
$$

for $p \in S=\partial D^{+}$and $x \in D$. The extension of the metrics can be chosen so that $F$ and $F^{\prime}$ are $C^{\infty}$-close to $F_{0}$.

We are going to construct a function $F^{\prime \prime}: S \times D \rightarrow \mathbb{R}$ which is also $C^{\infty}$-close to $F_{0}$ (so that there is a metric $\varphi^{\prime \prime}$ for which $F^{\prime \prime}$ is an enveloping function) and satisfies the following conditions:

(i) $\left.F^{\prime \prime}\right|_{S \times \partial D}=\left.F^{\prime}\right|_{S \times \partial D}$;

(ii) the resulting metric $\varphi^{\prime \prime}$ satisfies $\varphi^{\prime \prime} \geq \varphi$ pointwise.

The first condition implies that $\operatorname{vol}\left(D, \varphi^{\prime \prime}\right)=\operatorname{vol}\left(D, \varphi^{\prime}\right)$ by Lemma 2.2. The second one implies that $\operatorname{vol}\left(D, \varphi^{\prime \prime}\right) \geq \operatorname{vol}(D, \varphi)$. Thus the existence of such $F^{\prime \prime}$ implies the desired inequality $\operatorname{vol}\left(D, \varphi^{\prime}\right) \geq \operatorname{vol}(D, \varphi)$.

First we construct an auxiliary map $\Psi: U\left(D, \varphi^{\prime}\right) \rightarrow U(D, \varphi)$ between the unit tangent bundles of our metrics. This map sends every trajectory of the geodesic flow of $\varphi^{\prime}$ to the corresponding trajectory of the geodesic flow of $\varphi$ (with a linear change of time), where "corresponding" means that the associated geodesics of the two metrics connect the same pair of boundary points of $D$. More precisely, let $x \in D$ and $v \in U_{x}\left(D, \varphi^{\prime}\right)$, and let $\gamma^{\prime}:\left[0, T^{\prime}\right] \rightarrow D$ be the maximal $\varphi^{\prime}$-geodesic through $v$, that is, $\gamma^{\prime}\left(t^{\prime}\right)=x$ and $\dot{\gamma}^{\prime}\left(t^{\prime}\right)=v$ for some $t^{\prime} \in\left[0, T^{\prime}\right]$. Let $\gamma:[0, T] \rightarrow D$ be the $\varphi$-geodesic with the same endpoints on $\partial D$ : $\gamma(0)=\gamma^{\prime}(0)$ and $\gamma(T)=\gamma^{\prime}\left(T^{\prime}\right)$. 
If $T^{\prime} \neq 0$, define $\Psi(v)=\dot{\gamma}\left(t^{\prime} T / T^{\prime}\right)$, and if $T^{\prime}=0$ (or, equivalently, if $x \in \partial D$ and $v$ is tangent to $\partial D)$, let $\Psi(v)$ be the $\varphi$-unit vector positively proportional to $v$.

Clearly the map $\Psi$ defined this way is a homeomorphism between $U\left(D, \varphi^{\prime}\right)$ and $U(D, \varphi)$. Moreover $\Psi$ is a diffeomorphism and $\Psi$ goes to the identity as $\varphi, \varphi^{\prime} \rightarrow \varphi_{0}$ in $C^{\infty}$, see Proposition A.1 and Remark A.3 in Appendix A.

For every $p \in S$, define a map $G_{p}^{\prime}: D \rightarrow U\left(D, \varphi^{\prime}\right)$ by $G_{p}^{\prime}(x)=\operatorname{grad}_{\varphi^{\prime}} F_{p}^{\prime}(x)$. Note that $\pi \circ G_{p}^{\prime}=i d_{D}$ where $\pi: T^{*} D \rightarrow D$ denotes the bundle projection. Since $\Psi$ is close to the identity, the map

$$
H_{p}:=\pi \circ \Psi \circ G_{p}^{\prime}: D \rightarrow D
$$

is also $C^{\infty}$-close to the identity and hence is a diffeomorphism. Define a function $F_{p}^{\prime \prime}: D \rightarrow \mathbb{R}$ by $F_{p}^{\prime \prime}=F_{p}^{\prime} \circ H_{p}^{-1}$ and a function $F^{\prime \prime}: S \times D \rightarrow \mathbb{R}$ by $F^{\prime \prime}(p, x)=F_{p}^{\prime \prime}(x)$ for all $p \in S, x \in D$. Note that $F^{\prime \prime}$ depends continuously on $\varphi$ and $\varphi^{\prime}$ and $F^{\prime \prime}=F_{0}$ if $\varphi=\varphi^{\prime}=\varphi_{0}$, hence $F^{\prime \prime} \rightarrow F_{0}$ as $\varphi, \varphi^{\prime} \rightarrow \varphi_{0}$. Therefore, if $\varphi$ and $\varphi^{\prime}$ is sufficiently close to $\varphi_{0}$, there is a metric $\varphi^{\prime \prime}$ such that $F^{\prime \prime}$ is an enveloping function for it.

It remains to verify that $F^{\prime \prime}$ and $\varphi^{\prime \prime}$ satisfy the above conditions (i) and (ii). By construction, we have $\left.H_{p}\right|_{\partial D}=i d_{\partial D}$ for every $p \in S$, hence $\left.F_{p}^{\prime \prime}\right|_{\partial D}=\left.F_{p}^{\prime}\right|_{\partial D}$ and therefore $F^{\prime \prime}$ satisfies (i). To verify (ii), fix $x \in D \backslash \partial D$ and recall that the unit sphere of the dual norm $\varphi_{x}^{\prime \prime *}$ is parametrized by the family $\left\{d_{x} F_{p}^{\prime \prime}\right\}_{p \in S}$. Therefore

$$
\varphi^{\prime \prime}(v)=\sup _{p \in S}\left\{d_{x} F_{p}^{\prime \prime}(v)\right\}
$$

for every $v \in T_{x} D$. Let $v \in U_{x}(D, \varphi)$ and $\gamma:[0, T] \rightarrow D$ be the maximal $\varphi$-geodesic through $v$, that is, $x=\gamma\left(t_{0}\right)$ and $v=\dot{\gamma}\left(t_{0}\right)$ for some $t_{0} \in[0, T]$. Let $\gamma^{\prime}:\left[0, T^{\prime}\right] \rightarrow D$ be the $\varphi^{\prime}$-geodesic connecting the same endpoints on $\partial D$. Then, by the definition of $\Psi$, we have

$$
\Psi\left(\dot{\gamma}^{\prime}\left(t^{\prime}\right)\right)=\dot{\gamma}\left(t^{\prime} T / T^{\prime}\right)
$$

for all $t^{\prime} \in\left[0, T^{\prime}\right]$. Since $F^{\prime}$ is an enveloping function for $\varphi^{\prime}$, the $\varphi^{\prime}$-geodesic $\gamma^{\prime}$ is a gradient curve of the function $F_{p}^{\prime}:=F^{\prime}(p, \cdot)$ for some $p \in S$, that is,

$$
\dot{\gamma}^{\prime}\left(t^{\prime}\right)=G_{p}^{\prime}\left(\gamma^{\prime}\left(t^{\prime}\right)\right)
$$

for all $t^{\prime} \in\left[0, T^{\prime}\right]$. Therefore

$$
H_{p}\left(\gamma^{\prime}\left(t^{\prime}\right)\right)=\pi \circ \Psi \circ G_{p}^{\prime}\left(\gamma^{\prime}\left(t^{\prime}\right)\right)=\pi \circ \Psi\left(\dot{\gamma}^{\prime}\left(t^{\prime}\right)\right)=\gamma\left(t^{\prime} T / T^{\prime}\right)
$$

for all $t^{\prime} \in\left[0, T^{\prime}\right]$ or, equivalently,

$$
H_{p}^{-1}(\gamma(t))=\gamma^{\prime}\left(t T^{\prime} / T\right)
$$

for all $t \in[0, T]$. Thus

$$
F_{p}^{\prime \prime}(\gamma(t))=F_{p}^{\prime} \circ H_{p}^{-1}=F_{p}^{\prime}\left(\gamma^{\prime}\left(t T^{\prime} / T\right)\right)
$$

for all $t \in[0, T]$, hence

$$
d_{x} F_{p}^{\prime \prime}(v)=\left.\frac{d}{d t} F_{p}^{\prime \prime}(\gamma(t))\right|_{t=t_{0}}=\left.\frac{T^{\prime}}{T} \cdot \frac{d}{d t^{\prime}} F_{p}^{\prime}\left(\gamma^{\prime}\left(t^{\prime}\right)\right)\right|_{t^{\prime}=t_{0} T^{\prime} / T}=\frac{T^{\prime}}{T}
$$

since $\gamma^{\prime}$ is a $\varphi^{\prime}$-gradient curve of $F^{\prime}$ and therefore $\frac{d}{d t^{\prime}} F_{p}^{\prime}\left(\gamma^{\prime}\left(t^{\prime}\right)\right) \equiv 1$. Recall that $T=d_{\varphi}(a, b)$ and $T^{\prime}=d_{\varphi^{\prime}}(a, b)$ where $a=\gamma(0)=\gamma^{\prime}(0)$ and $b=\gamma(T)=\gamma^{\prime}\left(T^{\prime}\right)$, and $d_{\varphi^{\prime}}(a, b) \geq d_{\varphi}(a, b)$ since $b d_{\varphi^{\prime}} \geq b d_{\varphi}$. Therefore $d_{x} F_{p}^{\prime \prime}(v)=T^{\prime} / T \geq 1$.

This and (3.1) imply that $\varphi^{\prime \prime}(v) \geq 1$. Since $v$ is an arbitrary $\varphi$-unit tangent vector at $x$, and $x$ is an arbitrary interior point of $D$, it follows that $\varphi^{\prime \prime} \geq \varphi$. This completes the proof of (ii) and hence of Theorem 2 , 


\section{INJECTIVITY OF GEODESIC RAY TRANSFORM}

The goal of this section is to deduce Corollary 1 from Theorem 2 Let $\varphi$ be a simple Finsler metric on $D=D^{n}$ and $I_{\varphi}: C^{\infty}(D) \rightarrow C^{\infty}\left(\Gamma_{\varphi}\right)$ its geodesic ray transform. Let $f \in C^{\infty}(D)$ be such that $I_{\varphi} f=0$; we are to show that $f=0$.

For a small $\varepsilon>0$, define a Finsler metric $\varphi_{\varepsilon}$ on $D$ by

$$
\varphi_{\varepsilon}(v)=(1+\varepsilon f(\pi(v))) \cdot \varphi(v), \quad v \in T D
$$

where $\pi: T D \rightarrow D$ is the bundle projection. Let $p, q \in \partial D$ and $\gamma:[0, T] \rightarrow D$ the $\varphi$-geodesic connecting $p$ to $q$. Its $\varphi_{\varepsilon}$-length $L_{\varphi_{\varepsilon}}(\gamma)$ satisfies

$$
L_{\varphi_{\varepsilon}}(\gamma)=\int_{0}^{T}\left(1+\varepsilon f(\gamma(t)) d t=T+\varepsilon I_{\varphi} f(\gamma)=T\right.
$$

since $I_{\varphi} f=0$. Since $\varphi$ is simple, we have $T=L_{\varphi}(\gamma)=d_{\varphi}(p, q)$. Therefore

$$
d_{\varphi_{\varepsilon}}(p, q) \leq L_{\varphi_{\varepsilon}}(\gamma)=d_{\varphi}(p, q)
$$

Thus $b d_{\varphi_{\varepsilon}} \leq b d_{\varphi}$. Since $\varphi_{\varepsilon} \rightarrow \varphi$ in $C^{\infty}$ as $\varepsilon \rightarrow 0$, Theorem 2 applies and we conclude that $\operatorname{vol}\left(D, \varphi_{\varepsilon}\right) \leq \operatorname{vol}(D, \varphi)$ for a sufficiently small $\varepsilon$. On the other hand,

$$
\operatorname{vol}\left(D, \varphi_{\varepsilon}\right)=\int_{D}(1+\varepsilon f)^{n} d \operatorname{vol}_{\varphi}
$$

where $\operatorname{vol}_{\varphi}$ is the volume form of $\varphi$, therefore

$$
\int_{D}(1+\varepsilon f)^{n} d \operatorname{vol}_{\varphi} \leq \operatorname{vol}(D, \varphi) .
$$

The same argument applied to $-f$ in place of $f$ yields that

$$
\int_{D}(1-\varepsilon f)^{n} d \operatorname{vol}_{\varphi} \leq \operatorname{vol}(D, \varphi) .
$$

Summing these two inequalities we obtain

$$
\int_{D}\left((1+\varepsilon f)^{n}+(1-\varepsilon f)^{n}-2\right) d \operatorname{vol}_{\varphi} \leq 0
$$

This and the trivial inequality $(1+\varepsilon f)^{n}+(1-\varepsilon f)^{n}-2 \geq \varepsilon^{2} f^{2}$ for $n \geq 2$ imply that $\int_{D} f^{2} d \operatorname{vol}_{\varphi} \leq 0$, hence $f=0$. This completes the proof of Corollary 1 .

\section{Appendix A. Smoothness of $\Psi$}

The goal of this appendix is to prove the following technical fact used in the proof of Theorem 2 .

Proposition A.1. Let $\varphi$ and $\varphi^{\prime}$ be arbitrary simple Finsler metrics on $D=D^{n}$ and let a map $\Psi: U\left(D, \varphi^{\prime}\right) \rightarrow U(D, \varphi)$ be defined as in section 3. Then $\Psi$ is a $C^{\infty}$ diffeomorphism and it depends smoothly on $\varphi$ and $\varphi^{\prime}$.

Remark A.2. Technically, the domain $U\left(D, \varphi^{\prime}\right)$ of $\Psi$ is a variable (i.e., depending on $\varphi^{\prime}$ ) submanifold of $T D$. To formalize the notion of smooth dependence on $\varphi^{\prime}$ in Proposition A.1, one can identify all unit tangent bundles by means of the fiber-wise radial projection.

Remark A.3. Obviously $\Psi$ is the identity in the case when $\varphi^{\prime}=\varphi$. Therefore the smooth dependence on the metrics in Proposition A.1 implies that $\Psi$ goes to the identity (in $C^{\infty}$ ) as $\varphi, \varphi^{\prime} \rightarrow \varphi_{0}$. 
Proof of Proposition A.1. We write $\Psi=\Psi_{\varphi^{\prime}, \varphi}$ to emphasize the dependence on the metrics. We identify $D$ with the standard unit ball in $\mathbb{R}^{n}$ and denote by $\varphi_{e}$ the standard Euclidean metric (regarded as a Finsler metric on $D$ ). We will show that, for any simple metric $\varphi$, the map $\Psi_{\varphi, \varphi_{e}}$ is a diffeomorphism and it depends smoothly on $\varphi$. Proposition A.1 follows from this special case and the trivial identity $\Psi_{\varphi^{\prime}, \varphi}=\Psi_{\varphi_{e}, \varphi} \circ \Psi_{\varphi^{\prime}, \varphi_{e}}$.

For $u \in U D:=U(D, \varphi)$, let $\gamma_{u}$ denote the maximal $\varphi$-geodesic defined by the initial data $\dot{\gamma}(0)=u$ and let $\left[\tau^{-}(u), \tau^{+}(u)\right]$ be the domain of $\gamma_{u}$. Let $\ell(u)=$ $\tau^{+}(u)-\tau^{-}(u)$ be the length of the geodesic $\gamma_{u}, p^{ \pm}(u)=\gamma_{u}\left(\tau^{ \pm}(u)\right)$ its endpoints on the boundary, and $\tau(u)=\frac{1}{2}\left(\tau^{+}(u)+\tau^{-}(u)\right)$ the parameter of its midpoint. Define

$$
\begin{aligned}
\lambda(u) & =\frac{\left|p^{+}(u)-p^{-}(u)\right|}{\ell(u)} \in \mathbb{R}, \\
p(u) & =\frac{\left|p^{+}(u)+p^{-}(u)\right|}{2} \in D \subset \mathbb{R}^{n}, \\
w(u) & =\frac{p^{+}(u)-p^{-}(u)}{\left|p^{+}(u)-p^{-}(u)\right|} \in S^{n-1}
\end{aligned}
$$

if $u$ is not tangent to $\partial D$. If $u$ is tangent to $\partial D$, we have $\ell(u)=0$ and $p(u)=$ $p^{+}(u)=p^{-}(u)$ and extend $\lambda$ and $w$ by continuity: $\lambda(u)=|u|, w(u)=u /|u|$. Then the map $\Psi=\Psi_{\varphi, \varphi_{e}}$ can be written in the form

$$
\Psi(u)=(p(u)-\tau(u) \lambda(u) w(u), w(u)) \in D \times S^{n-1}=U\left(D, \varphi_{e}\right) .
$$

We are going to show that the functions $\tau, \lambda, p$ and $w$ (and hence $\Psi$ ) are smooth on $U D$. Let $V \subset U D$ denote the set of $\varphi$-unit vectors tangent to $\partial D$. This is a $(2 n-3)$-dimensional submanifold of the boundary $\partial U D$. The above functions are obviously smooth away from $V$, so we only need to prove their smoothness at $V$.

Extend the metric $\varphi$ to an open ball $D^{+} \supset D$. Since $\partial D$ is strictly convex with respect to $\varphi$, the trajectories of the geodesic flow are nowhere tangent to $V$. Therefore in a neighborhood of $V$ in $U D^{+}$there exists a coordinate system $(t, y, v)$ where $t, y \in \mathbb{R}$ and $v \in V$, such that the $t$-lines are trajectories of the geodesic flow and the points of $V$ have coordinates $(t, y, v)$ with $t=y=0$.

Observe that trajectories of the geodesic flow are tangent to $\partial U D$ at $V$. Hence, by the implicit function theorem, in a suitable neighborhood of $V$ the set $\partial U D$ is represented by a coordinate equation $y=h_{v}(t)=h(t, v)$ where $h \in C^{\infty}(\mathbb{R} \times V)$, and $h_{v}(0)=h_{v}^{\prime}(0)=0$. Since $\partial D$ is strictly convex, we have $h_{v}^{\prime \prime}(0) \neq 0$ and we may assume that $h_{v}^{\prime \prime}(0)>0$ (changing coordinate $y$ to $-y$ if necessary). Then the set $U D \subset U D^{+}$is locally the set of solutions of the inequality $y \geq h_{v}(t)$ in our coordinates. We need the following standard lemma.

Lemma A.4. Let $f \in C^{\infty}(\mathbb{R})$. Then

1. If $f(0)=0$, then there exists $g \in C^{\infty}(\mathbb{R})$ such that $f(x)=x g(x)$ for all $x \in \mathbb{R}$.

2. If $f$ is even, i.e. $f(x)=f(-x)$ for all $x \in \mathbb{R}$, then there exists $g \in C^{\infty}(\mathbb{R})$ such that $f(x)=g\left(x^{2}\right)$ for all $x \in \mathbb{R}$.

In both cases, $g$ depends smoothly on $f$.

Since $h_{v}(0)=h_{v}^{\prime}(0)=0$, we can apply the first part of Lemma A.4 to $h_{v}$ twice and conclude that $h_{v}(t)=t^{2} g_{v}(t)$ for some smooth function $g_{v}$. Observe that $g_{v}(0)=\frac{1}{2} h_{v}^{\prime \prime}(0)>0$, so $g_{v}$ is positive in a neighborhood of 0 . In this neighborhood 
we have $h_{v}(t)=f_{v}(t)^{2}$ where $f_{v}(t)=t \sqrt{g_{v}(t)}$, so $f_{v} \in C^{\infty}$ and $f^{\prime}(0)>0$. Let $u \in U D$ have coordinates $(t, y, v)$ where $t$ and $y$ are close to 0 . Then the values $\tau^{ \pm}(u)$ satisfy the equation

$$
h_{v}\left(t+\tau^{ \pm}(u)\right)=y
$$

since the point with coordinates $\left(t+\tau^{ \pm}(u), y, v\right)$ belongs to $\partial U D$. Therefore

$$
f_{v}\left(t+\tau^{ \pm}(u)\right)= \pm \sqrt{y}
$$

Since $f_{v}^{\prime}(0)>0, f_{v}$ is invertible near 0 and the above equation implies that

$$
\begin{aligned}
& \tau^{+}(u)=f_{v}^{-1}(\sqrt{y})-t, \\
& \tau^{-}(u)=f_{v}^{-1}(-\sqrt{y})-t,
\end{aligned}
$$

hence

$$
\tau(u)=\frac{1}{2}\left(f_{v}^{-1}(\sqrt{y})+f_{v}^{-1}(-\sqrt{y})\right)-t .
$$

The function $x \mapsto f_{v}^{-1}(x)+f_{v}^{-1}(-x)$ is defined in a neighborhood of 0 , smooth and even. Hence, by the second part of Lemma A.4 this function has a form $x \mapsto \theta_{v}\left(x^{2}\right)$ where $\theta_{v}$ is a smooth function (which depends smoothly on $v$ ). Then

$$
\tau(u)=\frac{1}{2} \theta_{v}(y)-t
$$

and therefore $\tau$ is smooth in a neighborhood of $V$.

To prove the smoothness of the map $p: U D \rightarrow D$, observe that

$$
\begin{aligned}
& p^{+}(u)=\pi\left(t+\tau^{+}(u), y, v\right)=\pi\left(f_{v}^{-1}(\sqrt{y}), y, v\right), \\
& p^{-}(u)=\pi\left(t+\tau^{-}(u), y, v\right)=\pi\left(f_{v}^{-1}(-\sqrt{y}), y, v\right)
\end{aligned}
$$

where $\pi$ is the bundle projection $U D \rightarrow D$ represented as a function of coordinates. Hence

Since the map

$$
p(u)=\frac{1}{2}\left(\pi\left(f_{v}^{-1}(\sqrt{y}), y, v\right)+\pi\left(f_{v}^{-1}(-\sqrt{y}), y, v\right)\right) .
$$

$$
(x, y, v) \mapsto \pi\left(f_{v}^{-1}(x), y, v\right)+\pi\left(f_{v}^{-1}(-x), y, v\right)
$$

is smooth and even with respect to the $x$ variable, by the second part of Lemma A.4 it has the form $(x, y, v) \mapsto \alpha\left(x^{2}, y, v\right)$ where $\alpha$ is a smooth function. Then $p(u)=\frac{1}{2} \alpha(y, y, v)$, hence $p$ is smooth.

To handle the functions $\lambda$ and $w$, we need the following lemma.

Lemma A.5. For every $f \in C^{\infty}(\mathbb{R})$ there exists $g \in C^{\infty}(\mathbb{R})$ such that

$$
f(\sqrt{y})-f(-\sqrt{y})=\sqrt{y} \cdot g(y)
$$

for all $y \geq 0$. Furthermore, $g$ depends smoothly on $f$.

Proof. Define $f_{1}(x)=f(x)-f(-x)$ for all $x \in \mathbb{R}$. Since $f_{1}$ is a smooth odd function, by the first part of Lemma A.4 it can be written in the form $f_{1}(x)=x f_{2}(x)$ where $f_{2}$ is an even smooth function (depending smoothly on $f$ ). By the second part of Lemma A.4 $f_{2}$ can be written in the form $f_{2}(x)=g\left(x^{2}\right)$ where $g$ is a smooth function (depending smoothly on $f_{2}$ and hence on $f$ ). Thus $f(x)=x g\left(x^{2}\right)$. Substituting $x=\sqrt{y}$ yields the result.

Lemma A.5 A.1 and A.2 imply that

$$
\ell(u)=\tau^{+}(u)-\tau^{-}(u)=f_{v}^{-1}(\sqrt{y})-f_{v}^{-1}(-\sqrt{y})=\sqrt{y} \tilde{\ell}(y, v)
$$

and

$$
p^{+}(u)-p^{-}(u)=\pi\left(f_{v}^{-1}(\sqrt{y}), y, v\right)-\pi\left(f_{v}^{-1}(-\sqrt{y}), y, v\right)=\sqrt{y} \tilde{w}(y, v)
$$


where $\tilde{\ell}$ and $\tilde{w}$ are smooth functions. Since $f_{v}^{-1}$ has nonzero derivative at 0 , we have $\tilde{\ell}(0, v) \neq 0$ and $\tilde{w}(0, v) \neq 0$. Now we have

$$
\lambda(u)=\frac{\left|p^{+}(u)-p^{-}(u)\right|}{\ell(u)}=\frac{|\tilde{w}(y, v)|}{\tilde{\ell}(y, v)}
$$

and

$$
w(u)=\frac{p^{+}(u)-p^{-}(u)}{\left|p^{+}(u)-p^{-}(u)\right|}=\frac{\tilde{w}(y, v)}{|\tilde{w}(y, v)|}
$$

where the right-hand sides are smooth in a neighborhood of the set $\{y=0\}$. Thus $\lambda$ and $w$ are smooth in a neighborhood of $V$ and therefore the map $\Psi=\Psi_{\varphi, \varphi_{e}}$ is smooth on $U D$.

It remains to verify that $\Psi$ is a diffeomorphism depending smoothly on the metric $\varphi$. It is easy to see from the definition that $\Psi^{-1}=\Psi_{\varphi_{e}, \varphi}$ is smooth away from the set $\Psi(V)$ of unit vectors tangent to $\partial D$. The strict convexity of the boundary easily implies that the derivative of $\Psi$ is non-degenerate at any point of $V$. Therefore $\Psi$ is a diffeomorphism. To verify the smooth dependence on $\varphi$, observe that the extension of $\varphi$ to $D^{+}$and the coordinates $(t, y, v)$ can be constructed in such a way that they depend smoothly on $\varphi$. Then all the smooth functions constructed throughout the proof depend smoothly on $\varphi$ and hence so does the map $\Psi$.

\section{REFERENCES}

[1] D. Bao, S.-S. Chern, Z. Shen, An introduction to Riemann-Finsler geometry. Graduate Texts in Mathematics 200, Springer-Verlag, New York, 2000, xx+431 pp.

[2] I. N. Bernstein, M. L. Gerver, A problem of integral geometry for a family of geodesics and an inverse kinematic seismics problem (Russian), Dokl. Akad. Nauk SSSR 243 (1978), no. 2, 302-305.

[3] I. N. Bernstein, M. L. Gerver, Conditions of distinguishability of metrics by godographs (Russian), Methods and Algoritms of Interpretation of Seismological Information, Computerized Seismology 13, Nauka, Moscow, 1980, pp. 50-73.

[4] G. Besson, G. Courtois and S. Gallot, Entropies et rigidités des espaces localement symétriques de courbure strictement négative, Geom. Funct. Anal., 5 (1995), 731-799.

[5] D. Burago and S. Ivanov, Boundary rigidity and filling volume minimality of metrics close to a flat one. Ann. of Math. (2) 171 (2010), no. 2, 1183-1211.

[6] D. Burago and S. Ivanov, Area minimizers and boundary rigidity of almost hyperbolic metrics, preprint, arXiv:1011.1570 2010.

[7] H. Busemann, Intrinsic area, Ann. of Math. (2) 48 (1947), 234-267.

[8] C. Croke, Rigidity and the distance between boundary points, J. Differential Geometry 33 (1991), 445-464.

[9] C. Croke, N. Dairbekov and V. Sharafutdinov, Local boundary rigidity of a compact Riemannian manifold with curvature bounded above, Trans. Amer. Math. Soc. 352 (2000), no. 9, 3937-3956.

[10] C. Croke and B. Kleiner, A rigidity theorem for simply connected manifolds without conjugate points, Ergodic Theory Dynam. Systems 18 (1998), no. 4, 807-812.

[11] M. Gromov, Filling Riemannian manifolds, J. Differential Geom. 18 (1983), 1-147.

[12] R. D. Holmes and A. C. Thompson, $N$-dimensional area and content in Minkowski spaces, Pacific J. Math. 85 (1979), 77-110.

[13] S. Ivanov, On two-dimensional minimal fillings, Algebra i Analiz 13 (2001), no. 1, 26-38 (Russian); St. Petersburg Math. J, 13 (2002), 17-25 (English).

[14] S. Ivanov, Filling minimality of Finslerian 2-discs, Proc. Steklov Inst. Math. 273 (2011), 176-190; arXiv:0910.2257

[15] S. Ivanov, Volume comparison via boundary distances, Proc. ICM 2010, vol.2, 769-784; arXiv:1004.2505

[16] H. Koehler, On filling minimality of simple Finsler manifolds, preprint, arXiv:1107.1650 v2, 2011. 
[17] R. Michel, Sur la rigidité imposeée par la longuer des géodésiques, Invent. Math. 65 (1981), 71-83.

[18] R. G. Mukhometov, On a problem of integral geometry over geodesics of a Riemannian metric (Russian), Conditionally correct mathematical problems and problems of geophysics, Collect. sci. Works, Novosibirsk 1979, 86-125 (1979).

[19] R. G. Muhometov, On a problem of reconstructing Riemannian metrics, Sibirsk. Mat. Zh. 22 (1981), no. 3, 119-135 (Russian); Siberian Math. J. 22 (1981), no. 3, 420-433 (English).

[20] L. Pestov and G. Uhlmann, Two-dimensional compact simple Riemannian manifolds are boundary distance rigid, Ann. of Math. (2) 161 (2005), 1093-1110.

[21] L. A. Santaló, Integral geometry and geometric probability, Encyclopedia Math. Appl., Addison-Wesley, London, 1976.

[22] V. A. Sharafutdinov, An inverse problem of determining the source in the stationary transport equation for a Hamiltonian system, Sibirsk. Mat. Zh. 37 (1996), no. 1, 211-235 (Russian); Siberian Math. J. 37 (1996), no. 1, 184-206 (English).

St. Petersburg department of V.A.Steklov Institute of Mathematics of the Russian Academy of Sciences, 191023, Fontanka 27, Saint Petersburg, Russia

E-mail address: svivanov@pdmi.ras.ru 